\title{
Improving Security using Honeyword for Online Banking Authentication System
}

\author{
Akshata Chor $^{1}$, Ashwini Gawali ${ }^{2}$, Ashwini Mohite ${ }^{3}$, Madhuri Tanpure ${ }^{4}$, Prof. P. B. Sahane ${ }^{5}$, Prof. P. B. Thorat ${ }^{6}$ \\ Department of Computer Engineering, P.K. Technical Campus, Chakan, Pune $e^{1,2,3,4,5,6}$
}

\begin{abstract}
In existing banking system, the only way to get access to the bank account is through the OTP which is not the secure way. If any unauthorized person stole your mobile and if that person guesses the password from user details then that person will get the access to the bank account easily. In propose system we are going to create and store honeywords in the honeypot, Honeywords are generate from the user details. Because of doing this if any unauthorized person will try to guess the password and if that guess password match with the honeypots words then alert for the legal user will generated and only login fail message will shows to that user. In this project we will use Honeyword Generation algorithm to achieve above goal.
\end{abstract}

Keywords: honeywords; honeypot; DoS attack; brute force attack; authentication

\section{INTRODUCTION}

Most data is digitized and stored in organizations' servers, threats (APT), corporate espionage, and other forms of from the user details. Because of doing this if any attacks are continuously on the rise. Revelation of unauthorized person will try to guess the password and if password files is a severe security problem that has that guess password match with the honeypots words then affected millions of users and banking applications, since leaked passwords make the users target of many possible cyber-attacks. These recent events have demonstrated that the weak password storage methods are currently in place on many web sites. In this study, we analyze the honeyword approach and give some remarks about the security of the system. Furthermore, we check out that the key item for this method is the generation algorithm of the honeywords such that they shall be unclear from the correct passwords. Therefore, we propose a new approach that uses passwords of users in the system for honeyword sets, i.e. realistic honeywords are provided. Moreover, this technique also reduces the storage cost compared with the honeyword method. Basically, a simple but clever idea behind the study is the insertion of false password called as honeywords associated with each users account. The cracked password files can be detected by the system administrator if a login attempt is done with a honeyword by the adversary. In our system we are going to create and store Honeywords. In the Honeypot because of doing this if any unauthorized person will try to guess the password and if that guess password match with the Honeypots words then alert for the legal user will generated and only login fail message will shows to that user.

\section{SYSTEM ARCHITRCHURE}

In existing system user is register on banking account using his personal details system is generated honeywords server site store on database. If user is login to account then system check password from honeywords. Password is correct then system sends OTP to authorized user. In alert for the legal user will generated and only login fail message will shows to that user. In this project we will use Honeyword Generation algorithm to achieve above goal. In this project, we scrutinize the honeyword system and present some remarks to highlight probable weak points.

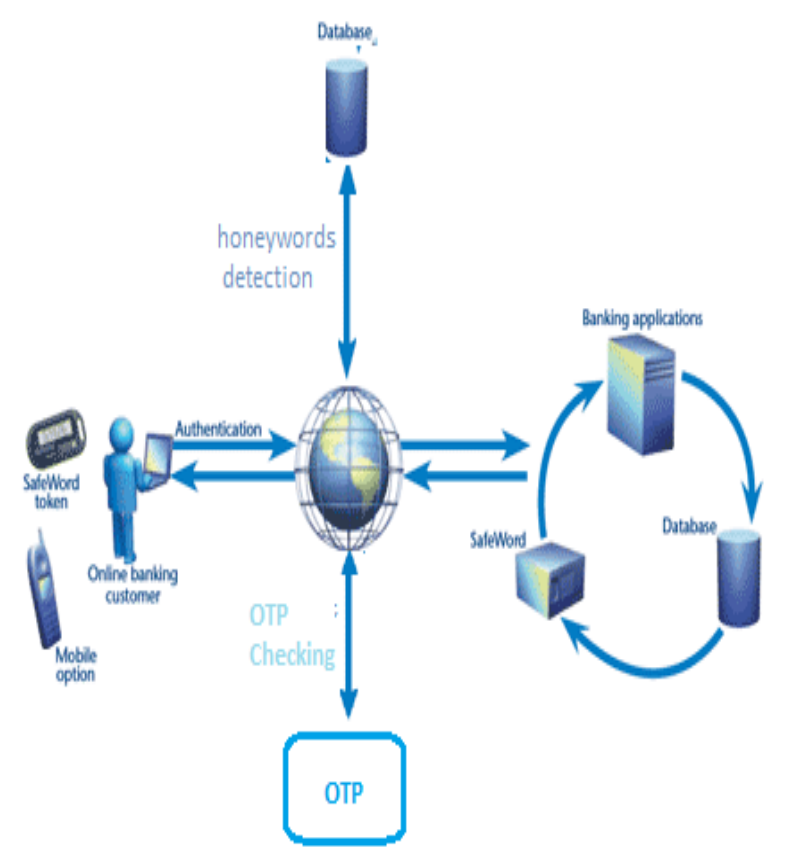

Also, we advise an alternative approach that selects the honeywords from existing user passwords in the management of system in order to provide realistic 
honeywords a perfectly flat honeyword generation method and also to reduce storage cost of the honeyword scheme.If attacker tries to access user account that time he/she enters any guess password. That times this password match with Honeywords on server site using pattern matching algorithm. This password match more than 50 percentage then system send alert message to authorized user. Then authorized user can change his/her password. Also user can understand attacker reach to his/her password.If Unauhorised user want to access to account then system check for honeyword in database system send email alert message to valid user that system also give MAC address and IP address of that system and temprory block account. if valid user want to use his/her account then he/she use account by hiting link which is get on mail.

\section{ALGORITHM}

Honeyword Generation Algorithm:-

1. Take User Information from user.

2. Calculate length of original password.

3. If honeyword count is even then take second half of original password go to step 5 else step 4 .

4. If honeyword count is odd then take first half of original password go to step 5 else step 3 .

5 . Concatenate the remaining half by randomly generated fake password from user information

6. If honeyword count is $n$ then go to step 7 else go to step 3.

7. Stop.

\section{LITERATURE SURVEY}

1.H. Bojinov, E. Bursztein, X. Boyen, and D. Boneh,Kamouflage: Loss-resistant password management. In this paper described a new architecture for building theft-resistant password managers. An attacker who steals a laptop or cell phone with a Kamouflage-based password manager is forced to carry out a considerable amount of online work before obtaining any user credentials.

2. P. G. Kelley, S. Komanduri, M. L. Mazurek, R. Shay, T. Vidas, L.Bauer, N. Christin, L. F. Cranor, and J. Lopez, Guess again (and gain and again): Measuring password strength by simulating password-cracking algorithms. In this paper described Text-based passwords remain the dominant authentication method in computer systems, despite significant advancement in attacker's capabilities to perform password cracking. In response to this threat, password composition policies have grown increasingly complex. However, there is insufficient research defining metrics to characterize password strength and using them to evaluate password-composition policies.

3. K. Brown, The dangers of weak hashes. In this paper described the basics of password hashing, look at password cracking software and hardware, and discuss best practices for using hashes securely.
4. M. Weir, S. Aggarwal, B. de Medeiros, and B. Glodek, Password cracking using probabilistic context-free grammars. In this paper described choosing the most effective word-mangling rules to use when performing a dictionary-based password cracking attack can be a difficult task.

5. F. Cohen, The use of deception techniques: Honeypots and decoys. In this paper described honeypots and similar sorts of decoys, discuss their historical use in defense of information systems, and describe some of their uses today. We will then go into a bit of the theory behind deceptions, discuss their limitations, and put them in the greater context of information protection.

\section{RESULT}

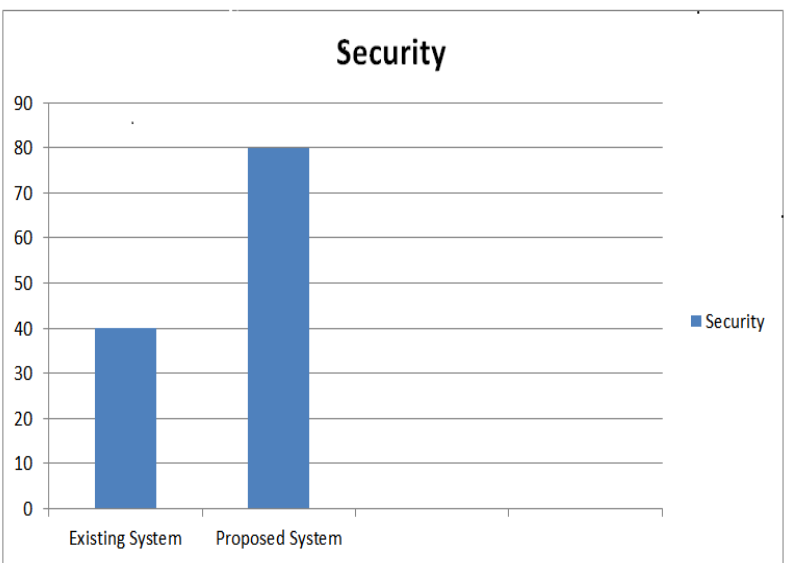

VI. CONCLUSION AND FUTURE SCOPE

Conclusion: In this project, we have analyzed the surety of the honeyword system and addressed a number of flaws that need to be handled before successful fulfillment of the scheme. In this, we have pointed out that the potency of the honeyword system depends on the honeyword generation algorithm, i.e. flatness of the generator algorithm find out the chance of distinguishing the correct password out of respective sweet words. This project will consist of security to the user account through text password and the OTP. The modules of our project to provide security using Honeyword Generation Algorithm and a way to motivate authorized user to continue provide security for user account.

Future Scope: - We will find out location of unauthorized user using GPS and it will make system more secure.

\section{ACKNOWLEDGMENT}

We might want to thank the scholar and also distributers for making their assets available. We additionally selective to commentator for their consequence advice furthermore thank the school powers for giving the obliged base and backing. 


\section{REFERENCES}

[1] A. Pathak, "An analysis of various tools, methods and systems to generate fake accounts for social media," Ph.D. dissertation, Northeastern University Boston, Boston, MA, USA, 2014.

[2] D. Mirante and C. Justin, "Understanding password database compromises," Dept. of Comput. Sci. Eng. Polytechnic Inst. of NYU, New York, NY, USA: Tech. Rep. TR-CSE-2013-02, 2013.

[3] K. Brown, "The dangers of weak hashes," SANS Institute InfoSec Reading Room, Maryland US, pp. 1-22, Nov. 2013.

[4] M. H. Almeshekah, E. H. Spafford, and M. J. Atallah, "Improving security using deception," Center for Education and Research Information Assurance and Security, Purdue Univ., West Lafayette, IN, USA: Tech. Rep. CERIAS Tech. Rep.2013-13, 2013.

[5] A. Juels and R. L. Rivest, "Honeywords: Making passwordcrackingdetectable,"in Proc. ACM SIGSAC Conf. Comput.Commun. Security, 2013, pp. 145-160.

[6] M. Burnett. The pathetic reality of adobe password hints.

[7] Z. A. Genc, S. Kardas, and M. S. Kiraz, "Examination of a new defense mechanism: Honeywords," IACR Cryptology ePrint Archive, Report 2013/696, 2013.

[8] L. Zhao and M. Mannan, "Explicit authentication response considered harmful," in Proc. Workshop New Security Paradigms Workshop, 2013, pp. 77-86.

[9] J. Bonneau, "The science of guessing: Analyzing an anonymized corpus of 70 million passwords," in Proc. IEEE Symp. Security Privacy, 2012, pp. 538-552.

[10] D. Malone and K. Maher Investigating the distribution of password choices. In Proc. $21^{\text {st }}$ Int. Conf. World Wide Web, 2012, pp. 301310 . 